\title{
La competencia de liderazgo en el grado de enfermería: Un análisis factorial de componentes principales
}

\author{
The leadership competence in Nursing Studies: a principal components \\ factor analysis
}

\section{Nuria Rodríguez-Ávila ${ }^{a}$, Carme Riera i Prunera ${ }^{\mathrm{b}}$, Carmen Moreno-Arroyo ${ }^{\mathrm{c}}$, Teresa $\mathbf{M}^{\mathrm{a}}$ Monllau-Jaques ${ }^{\mathrm{d}}$, Montserrat Puig-Llobet ${ }^{\mathrm{e}}$}

\author{
${ }^{a}$ Departamento de Sociología \\ Facultad de Economía y Empresa \\ Universidad de Barcelona \\ Avda. Diagonal 696, 08034, Barcelona \\ ${ }^{\mathrm{b}}$ Departamento Econometría, Estadística y Economía Aplicada \\ Facultad de Economía y Empresa \\ Universidad de Barcelona \\ Avda. Diagonal 690, 08034, Barcelona \\ ${ }^{c}$ Departamento de Enfermería Fundamental y Medicoquirúrgica \\ Escola Universitària d'Infermeria, Facultat de Medicina i Ciències de la Salut \\ Universidad de Barcelona \\ C/Feixa Llarga, s/n, 08907, l’Hospitalet de Llobregat \\ ${ }^{\mathrm{d}}$ Departamento de Economía y Empresa \\ Universitat Pompeu Fabra \\ C/Ramon Trias Fargas, 25-27, 08005, Barcelona \\ ${ }^{\text {e }}$ Departamento de Enfermería de Salud Pública, Salud Mental y Maternoinfantil \\ Escola Universitària d'Infermeria, Facultat de Medicina i Ciències de la Salut \\ Universidad de Barcelona \\ C/Feixa Llarga, s/n, 08907, l'Hospitalet de Llobregat \\ nrodriguez@ub.edu mcriera-prunera@ub.edu carmenmoreno@ub.edu \\ teresa.monllau@upf.edu monpuigllob@ub.edu
}

\begin{abstract}
Resumen
El aspecto competencial está cada vez más presente en la sociedad, desde los niveles académicos a los laborales. De una buena base en la adquisición de competencias depende en gran medida el éxito profesional de los graduados, de ahí que el análisis para potenciar su adquisición se haya convertido en un tema de estudio recurrente, tanto en lo que respecta a las competencias básicas/transversales, como a las específicas. Estas últimas serán las que tendrán una incidencia distinta en función del tipo de estudio, a la vez que experimentarán variaciones importantes en función de los retos y proyectos innovadores que las organizaciones deban afrontar. Entre las primeras, una de las competencias que desempeña un papel primordial, y en la que se ha centrado el presente estudio, es la capacidad de liderazgo. En concreto, este trabajo analiza cómo perciben y adquieren la capacidad de liderazgo los estudiantes de enfermería de la Universidad de Barcelona, dado que les resulta fundamental para ejercer la influencia en un grupo y para lograr las metas propuestas.
\end{abstract}

Para ello se preparó un cuestionario con 70 preguntas que recogían las competencias transversales y específicas adquiridas en el proceso de aprendizaje, así como la actitud del estudiante ante el proceso de aprendizaje. La población sujeto de estudio fueron los estudiantes del grado de enfermería de la Universidad de Barcelona durante el curso académico 2012-2013; y la técnica de muestreo seleccionada fue la del muestreo incidental. 
El trabajo aplica el análisis multivariante para reducir la dimensionalidad de las variables estudiadas. En concreto, el análisis factorial mediante componentes principales ha permitido identificar los aspectos que resumen y agrupan las 20 variables estudiadas en 4 factores: capacitación para la profesionalización, trabajo autónomo, capacidad crítica y toma de decisiones en equipos. Posteriormente, a partir de las puntuaciones de estos cuatro factores y con la inclusión de las variables edad, sexo y procedencia, se construyó un modelo de regresión logístico multinomial. Los resultados obtenidos indican que los cuatro factores influyen positivamente a la hora de incrementar la probabilidad de pasar de una capacidad de liderazgo de un nivel nulo a un nivel medio, mientras que en el incremento de la probabilidad del paso de una capacidad de liderazgo nula a una de elevada sólo los tres últimos factores tienen influencia.

Palabras clave: Competencias, Liderazgo, Análisis factorial, Enfermería.

\begin{abstract}
Jobs are increasingly influenced by the empowerment of competencies allowing organizations to face the challenges and innovative projects presented to them, as well as addressing problem solving in the best possible way. In this sense, one of the competencies that plays a major role is leadership. In this paper, we want to analyse how nursing students at the University of Barcelona perceive and acquire the leadership capacity, since it is fundamental to influence a group and to achieve the required goals. In the specific case of the nursing profession, which is strongly influenced by the organizational changes of the Health Systems, the management of nursing services is considered one of the most relevant aspects in the health organization chart (CODEM, 2016).
\end{abstract}

The population of study comprised the nursing degree students during the academic year 2012-2013 at the University of Barcelona. The sampling technique selected was a non-probabilistic sampling for convenience, also known as incidental sampling (Buendía, 1994). This technique is chosen given the accessibility and proximity of the sampling subjects (i.e. students attending class on the day the questionnaire was provided).

The questionnaire used in this study consists of 70 questions covering all basic and specific competences acquired in the learning process, as well as the student's attitude towards the learning process, as it is explained in the Tuning project. Nevertheless, we have adapted it to the nursing degree. Questions are grouped into four blocks. The first corresponds to sociodemographic aspects, the second has to do with the assessment of skills, the third includes the acquisition of transversal competences such as leadership capacity, problem solving ability or teamwork. Finally, the fourth block refers to attitudes when it comes to studying. The variables studied were measured on a Likert scale from 1 to 4 , where 4 indicates a high degree of acquisition. The Cronbach's alpha value for questionnaire reliability is 0.925 , showing, therefore, high reliability. The number of participants was 171.

In the study we used a multivariate analysis and a multinomial regression to explore the results. The multivariate analysis allows us to reduce the dimensionality of the variables under study. More specifically, the factor analysis using principal components allowed us to identify 4 factors that summarize and group the 20 variables studied; namely (from more to less weight): training for professionalization, self-employment, critical capacity and team decision-making.

Secondly, a Multinomial Regression has been elaborated using the factorial scores to analyse in depth the leadership aspect. In particular, it has been found that all four factors exert a positive influence when moving from a null leadership capacity to a medium capacity, whereas in the transition from a null leadership capacity to a high one only the last three factors seem to have some influence.

Key words: Competences, Leadership, Factor analysis, Nursing studies.

\title{
1.Introducción
}

Las competencias y el conjunto de habilidades que una persona debe desarrollar en el despeño de su actividad profesional es uno de los aspectos en los que actualmente se centran muchos estudios académicos, aunque no se trata de un tema que ocupe exclusivamente al ámbito docente, sino que, por su 
importancia, trasciende más allá de este (Alcañiz, Claveria, y Riera-Prunera, 2014), (Alcañiz, RieraPrunera, y Claveria, 2013). Un aspecto importante es que la utilización y aplicación de la gestión por competencias ofrece un nuevo estilo de dirección en el seno de las organizaciones, en las que el factor humano es clave. En el entorno académico se ha hecho hincapié en cómo debe ser el proceso de adquisición, observándose qué debe ser lo aprendido, y evaluado, y cómo puede ser mejorado y transferido. A este respecto, uno de los trabajos más consultados es el de González y Wagenaar, que define las competencias a partir de la visualización de los perfiles profesionales y académicos de una titulación, incorporando nuevos procesos de docencia y gestión del conocimiento, a la vez que introduciendo flexibilidad y organización del aprendizaje, y, por último introduciendo un lenguaje adecuado para el intercambio entre graduados y profesionales (González y Wagenaar, 2003).

A nivel profesional, los puestos de trabajo vienen cada vez más marcados por la potenciación de las competencias de manera que permita a las organizaciones afrontar los retos y proyectos innovadores que se les presenten, así como encarar la resolución de problemas de la mejor manera posible. Para ello una de las competencias que desempeña un papel primordial es la capacidad de liderazgo (Cortés-González, Hernández-Saavedra, Marchena-Rivera, Marqueti-Machado, y Nava-Galán, 2012). En este trabajo queremos analizar cómo perciben y adquieren la capacidad de liderazgo los estudiantes de enfermería de la Universidad de Barcelona, dado que es fundamental para influir en un grupo y para lograr las metas. En el caso concreto de la profesión de enfermería, que está envuelta en los cambios organizacionales de los Sistemas Sanitarios, la gestión de servicios de Enfermería se presenta como uno de los aspectos más relevantes en el organigrama de la salud (CODEM, 2016).

Según Cortes González (Cortés-González et al., 2012), el estilo de liderazgo viene determinado por una combinación entre orientación a la producción y focalización en los empleados a través de las relaciones inter-personales. Las cualidades que caracterizan a un líder pueden ser fomentadas a partir de ciertas competencias y habilidades que se pueden adquirir en el proceso académico. En este sentido, la profesión de Enfermería debe formar profesionales con capacidad de liderar en todos los aspectos y niveles de la organización sanitaria. Esta capacidad de liderar viene relacionada con sus habilidades de colaboración de alto nivel siempre en pro de la mejora de los cuidados a los pacientes. Los puestos que suele ocupar un/a enfermero/a le lleva a actividades de coordinación en algunas ocasiones, así como de integración y facilitador de las prácticas o procedimientos de cuidados a los pacientes. Por eso los profesionales de enfermería deben tener habilidades vinculadas a los puestos de trabajo, que les permitan facilitar, diseñar y poner en práctica las estrategias de cambio a la hora de proporcionar los cuidados. El desarrollo de estas capacidades de liderazgo es fundamental para avanzar en la profesión y en el ejercicio profesional. De ahí que el grado tenga como uno de sus ejes fomentar la capacidad de liderazgo desde lo académico a la práctica clínica.

Este trabajo tiene en cuenta dimensiones como habilidades en el ejercicio de la profesión, habilidades para el aprendizaje y sobretodo en las formas de estudio que se emplean; considerando que las competencias del grado son esenciales en el aprendizaje del estudiante (UB, 2014). De hecho, en la última década los estudios de grado se han adaptado a normativa europea a través de la implementación de competencias en las diferentes asignaturas que lo conforman. Ello se ha hecho considerando las competencias diseñadas por distintos proyectos nacionales e internacionales, entre los que cabe destacar uno de los pioneros, el proyecto Tuning, y con el fin de incorporar las necesidades tanto del sector privado como del sector público, en muchos casos discrepantes. Son numerosos los estudios que se han ocupado del análisis de tales discrepancias en aras a reducirlas para así mejorar el encaje entre lo que la universidad ofrece y lo que el mundo laboral demanda (Paadi, 2014), (Freire, Teijeiro Álvarez, y Pais Montes, 2013), (Teijeiro, Rungo, y Freire, 2013), (Alcañiz et al., 2013), (Martín Del Peso, RabadánGómez, y Hernández-March, 2013), (Mason, Williams, y Cranmer, 2009), (Navarro, Iglesias, y Torres, 2006). 


\section{Estudio del caso: grado de enfermería en la Universidad de Barcelona}

El Título de Grado de Enfermería de la Universidad de Barcelona (UB) tienen como objetivo capacitar para el ejercicio de la profesión de forma general, con formación científica y actitud crítica, reflexiva y humanista para valorar, identificar, actuar y evaluar las necesidades de salud y de cuidados de las personas, las familias y la comunidad (UB, 2014). La primera promoción de Grado de Enfermería de la Universidad de Barcelona se inició en el curso académico 2009-2010. Las competencias que forman parte del plan de estudios del Título de Grado de Enfermería de la UB están regidas por las competencias profesionales definidas por la ANECA para los profesionales enfermeros y las definidas en el proyecto Tuning, tal y como se especifica en la Tabla 1.

\section{Básicas}

CB1. Poseer y comprender conocimientos básicos

CB2. Aplicar los conocimientos a su trabajo o vocación de una forma profesional

CB3. Capacidad de reunir e interpretar datos relevantes

CB4. Habilidad para transmitir información, ideas, y soluciones a problemas

CB5. Habilidad de aprendizaje con alto grado de autonomía

\section{Generales}

CG1. Capacidad creativa y emprendedora según actitudes

CG2. Capacidad de aprendizaje y responsabilidad

CG3. Trabajo en equipo

CG4. Capacidad creativa y emprendedora según habilidades

CG5. Sostenibilidad

CG6. Capacidad comunicativa

Fuente: Elaboración propia a partir de La Memoria para la Verificación del Título de Grado de Enfermería de la UB.

Tabla 1: Competencias básicas y generales en el Grado de enfermería UB

Por lo que respecta a las competencias específicas, se estructuran en los siguientes bloques: práctica profesional, ética y legal; prestación de cuidados; gestión de cuidados; y desarrollo profesional (MorenoArroyo, 2015). A su vez, cada bloque contempla diferentes elementos que le otorgan un significado más concreto, tal y como recoge la Tabla 2.

$\begin{array}{cl}\text { Práctica profesional, ética } \\ \text { CE1. } & \text { Responsabilidad } \\ \text { CE2. } & \text { Práctica ética } \\ \text { CE3. } & \text { Práctica legal } \\ & \\ & \\ \text { Prestación de cuidados } \\ \text { CE } 4 . & \text { Valoración } \\ \text { CE 5. } & \text { Planificación } \\ \text { CE 6. } & \text { Ejecución } \\ \text { CE 7. } & \text { Evaluación }\end{array}$

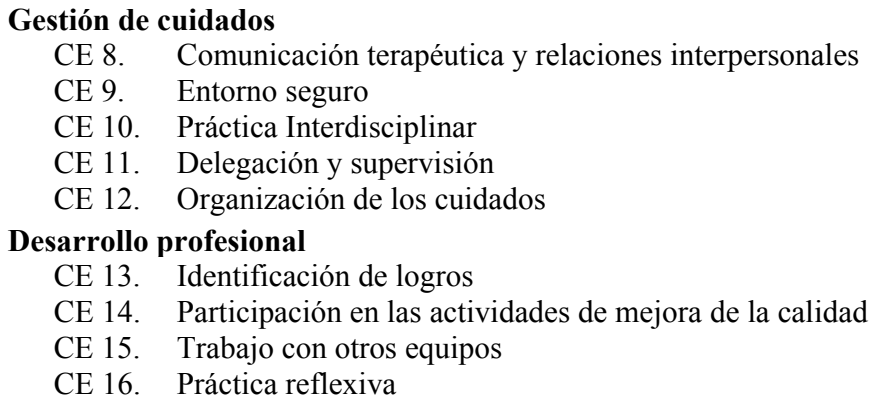

Fuente: elaboración propia a partir de la Memoria para la verificación del Título de Grado de Enfermería de la UB.

Tabla 2: Competencias específicas del Grado Enfermería Universidad de Barcelona 


\section{Metodología}

\subsection{Muestra}

La población sujeto de estudio han sido los estudiantes de primer curso del grado de enfermería de la Universidad de Barcelona, campus de L'Hospitalet de Llobregat, durante el curso el curso académico 2012-2013, para la asignatura de Ciencias psicosociales aplicadas a la salud del grado de enfermería, de carácter fundamental y equivalente a 9 créditos ECTS. La técnica de muestreo seleccionada fue la de muestreo no probabilístico por conveniencia o también conocida como muestreo incidental ${ }^{1}$ (Buendía, 1994). Se escogió esta técnica dada la accesibilidad y proximidad de los sujetos seleccionados por los asistentes a clase el día que se suministró el cuestionario.

\subsection{Instrumento}

El cuestionario utilizado para este estudio consta de 70 preguntas que recogen todas las competencias tanto transversales como específicas adquiridas en el proceso de aprendizaje, así como la actitud que tiene el estudiante ante dicho proceso, tal y como queda recogido en el proyecto Tuning, aunque adaptado al estudio sobre el grado de enfermería. Dicho instrumento consta de cuatro bloques. El primero corresponde a aspectos sociodemográficos, el segundo tiene que ver con valoración de las habilidades, el tercero está en relación con la adquisición de competencias transversales (i.e. capacidad de liderazgo, capacidad de resolución de problemas, trabajo en equipo...). Por último, el cuarto bloque es el referido a actitudes a la hora de estudiar. Las variables estudiadas se han medido en una escala de Likert de 1 a 4, donde 4 indica un grado elevado de adquisición.

El valor del alfa de Cronbach para la fiabilidad de cuestionario es 0,925 , indicando una elevada fiabilidad. Al finalizar el semestre se les suministró el cuestionario con una respuesta de 171 casos de los cuales el $83 \%$ fueron mujeres y el 17\%, hombres. El número de casos incluidos finalmente fue de 161, $94,2 \%$ del total de la muestra. Las edades van desde los 18 años a los 48 siendo la media de años de 21,34, con una desviación de 5,4 años.

La metodología aplicada en este trabajo utiliza el análisis multivariante y los modelos de regresión logit multinomial. En primer lugar, a partir de los datos recogidos, se ha realizado un análisis factorial mediante componentes principales que nos permite reducir la dimensionalidad de los datos y agrupar los 20 aspectos estudiados de manera homogénea. El objetivo es encontrar el mínimo número de dimensiones que expliquen la máxima información contenida en los datos. Posteriormente, a partir de la información de los factores extraídos, se ha construido una regresión logit multinomial para analizar en profundidad el liderazgo en enfermería.

\subsection{Análisis Factorial mediante componentes principales}

La prueba de medida de Kaiser-Meyer-Olkin (KMO) ofrece un valor de 0,895 , por lo que rechazamos la hipótesis nula que establece que la matriz de correlaciones sea igual a la matriz identidad, y por tanto, existe correlación entre las variables; en consecuencia tiene sentido agruparlas en factores. Se incorporan un total de 20 variables que finalmente se reagrupan en los 4 factores extraídos con autovalores ${ }^{2}$ superiores a la unidad que permiten explicar el 57,09\% del total de la varianza de los datos. En concreto, en el Gráfico 1 de sedimentación se puede apreciar que los autovalores de las cuatro primeras componentes son mayores que la unidad, y por tanto serán éstas las que se han utilizado para resumir la información de las variables estudiadas.

\footnotetext{
${ }^{1}$ En Buendía, "que es aquel que realiza el investigador aprovechando los elementos de la población que le son fácilmente accesibles" (Buendía, 1994).

${ }^{2}$ Los autovalores expresan la cantidad de varianza total explicada por cada factor.
} 


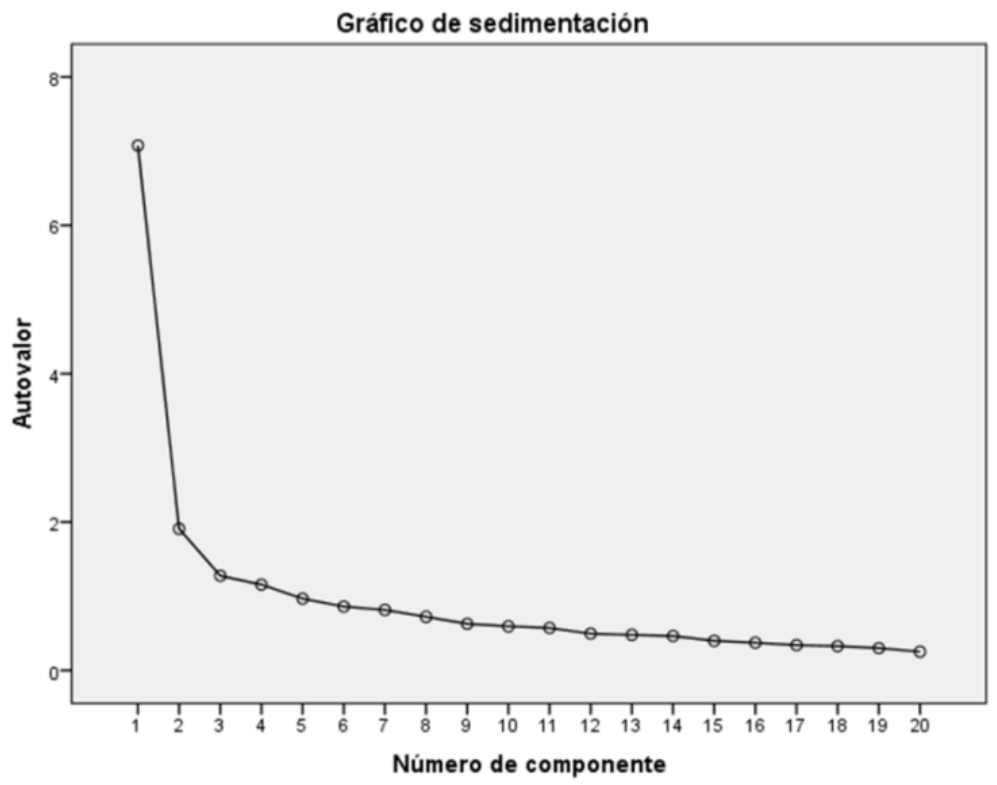

Fuente: Elaboración propia a partir de SPSS.

Gráfico 1: Sedimentación: estudio de componentes

El primer factor, que recoge una tercera parte de la variabilidad total, está compuesto por 5 ítems que reflejan lo que hemos llamado la capacitación para la profesionalización. Este factor, que recoge un $35 \%$ de la variancia, mide elementos básicos para el ejercicio profesional, que van desde los esenciales de la profesión de enfermería, el nuevo aprendizaje y la puesta en práctica del mismo, así como la capacidad de analizar situaciones y sintetizar información para poder planificar actuaciones futuras. El segundo factor, que incluye también 5 ítems y explica alrededor de un $10 \%$ de la varianza, refiere al trabajo autónomo, dado que mide la capacidad de diseñar y gestionar el tiempo de trabajo, incorporando elementos como la capacidad de expresión oral y el uso del lenguaje en el proceso de comunicación. La capacidad de trabajo autónomo permite medir cómo se organiza el profesional para la realización de las tareas y cómo éste después lo transmite y comunica. El tercer factor, con 6 aspectos y un $6 \%$ de la varianza, representaría la capacidad de crítica y comunicación, dado que incorpora dimensiones como el pensamiento crítico, la capacidad de comunicarse, o la responsabilidad y el compromiso con la ética profesional. Por último, el cuarto factor recoge 4 aspectos que representan cerca del $6 \%$ de la variabilidad total y podría decirse que refleja la capacidad de toma de decisiones y trabajo en equipo al contar con las variables relacionadas con la toma de decisiones ante situaciones complejas, el trabajo con otros colegas y grupos interdisciplinares, la actuación en situaciones donde estén presentes problemas a resolver, y la capacidad de generar nuevas soluciones e ideas (Tablas 3 y 4 ).

\begin{tabular}{|c|c|c|c|c|c|c|c|c|c|}
\hline \multirow[t]{2}{*}{ Componente } & \multicolumn{3}{|c|}{ Autovalores iniciales } & \multicolumn{3}{|c|}{$\begin{array}{l}\text { Sumas de extracción } \\
\text { de cargas al cuadrado }\end{array}$} & \multicolumn{3}{|c|}{$\begin{array}{c}\text { Sumas de rotación } \\
\text { de cargas al cuadrado }\end{array}$} \\
\hline & Total & $\begin{array}{c}\% \text { de } \\
\text { varianza } \\
\end{array}$ & $\begin{array}{c}\% \% \\
\text { acumulado }\end{array}$ & Total & $\begin{array}{c}\% \text { de } \\
\text { varianza } \\
\end{array}$ & $\begin{array}{c}\% \% \\
\text { acumulado }\end{array}$ & Total & $\begin{array}{c}\% \text { de } \\
\text { varianza } \\
\end{array}$ & $\begin{array}{c}\% \\
\text { acumulado }\end{array}$ \\
\hline 1 & 7,076 & 35,382 & 35,382 & 7,076 & 35,382 & 35,382 & 3,451 & 17,256 & 17,256 \\
\hline 2 & 1,909 & 9,543 & 44,925 & 1,909 & 9,543 & 44,925 & 2,936 & 14,679 & 31,935 \\
\hline 3 & 1,276 & 6,380 & 51,305 & 1,276 & 6,380 & 51,305 & 2,760 & 13,799 & 45,734 \\
\hline 4 & 1,156 & 5,779 & 57,084 & 1,156 & 5,779 & 57,084 & 2,270 & 11,351 & 57,084 \\
\hline
\end{tabular}

Fuente: Elaboración propia.

Tabla 3: Análisis de componentes principales. Varianza total explicada. 


\begin{tabular}{lrrrr}
\hline & \multicolumn{5}{c}{ Componente } \\
\cline { 2 - 6 } & $\mathbf{1}$ & $\mathbf{2}$ & $\mathbf{3}$ & $\mathbf{4}$ \\
\hline Factor 1: Capacitación profesional & & & & \\
Conocimientos básicos de la profesión &, 723 &, 219 &, 125 &, 086 \\
Capacidad de aplicar conocimientos a la práctica &, 708 &, 017 &, 279 &, 141 \\
Capacidad de análisis y síntesis &, 697 &, 085 &, 142 &, 005 \\
Planificación y gestión del tiempo &, 696 &, 225 &,- 036 &, 364 \\
Capacidad de aprendizaje &, 631 &, 272 &, 298 &, 248 \\
\hline Factor 2: Trabajo autónomo & & & & \\
Diseño y gestión de proyectos &, 168 &, 761 &, 044 &, 049 \\
Habilidad para trabajar de manera autónoma &, 180 &, 699 &,- 051 &, 317 \\
Iniciativa y espíritu emprendedor &, 053 &, 654 &, 303 &, 131 \\
Habilidad para trabajar en un contexto internacional &, 004 &, 589 &, 426 &, 116 \\
Comunicación oral y escrita en la propia lengua &, 363 &, 552 &, 247 &, 072 \\
\hline Factor 3: Critica y comunicación & & & & \\
Capacidad crítica y autocrítica &, 279 &, 057 &, 730 &, 057 \\
Compromiso ético &,- 045 &, 389 &, 669 &, 289 \\
Pensamiento crítico &, 195 &, 315 &, 523 &, 223 \\
Capacidad de comunicarse &, 415 &, 278 &, 518 &, 172 \\
Capacidad de adaptación a nuevas situaciones &, 423 &,- 139 &, 483 &, 389 \\
Conocimiento de una segunda lengua &, 195 &, 355 &, 414 &,- 075 \\
\hline Factor 4: Toma de decisiones y trabajo en equipo & & & & \\
$\quad$ Toma de decisiones &, 285 &, 046 &, 328 &, 671 \\
Trabajo en equipo &, 316 &, 225 &,- 010 &, 643 \\
Capacidad de generar nuevas ideas (creatividad) &,- 088 &, 277 &, 090 &, 621 \\
Resolución de problemas &, 408 &,- 047 &, 417 &, 580 \\
\hline$\quad$ Fuente: Elaboración propia a partir del estudio VIOPES & & & &
\end{tabular}

Tabla 4: Matriz de componentes rotados. Método de extracción: análisis de componentes principales. Método de rotación: Varimax con normalización Kaiser (la rotación ha convergido en 11 iteraciones).

\subsection{El modelo de regresión multinomial}

Tras la identificación de los factores, continuamos el análisis para ver en qué medida éstos influyen en la capacidad de liderazgo. Con este fin se realizó una regresión logística multinomial incorporando como variables independientes la edad del estudiante, el género $(1=$ Hombre, $2=$ Mujer $)$ y la procedencia (1=Bachillerato $2=$ Formación Profesional), junto con los cuatro factores que han sido obtenidos a partir del análisis de componentes principales. El estadístico de razón de verosimilitud para el contraste de significación conjunta indica que el modelo es globalmente significativo ${ }^{3}$. Por lo que respecta a la bondad de ajuste, el pseudo- $\mathrm{R}^{2}$ de $\mathrm{McFadden}^{4}$ arroja un valor de 0,217; la prueba de Cox y Snell, 0,346; y la prueba de Nagelkerke, 0,403, con lo que puede hablarse de un ajuste más bien bueno. Además, el modelo construido predice de forma correcta un $68,9 \%$ de los casos, con especial acierto en el pronóstico de la capacidad de liderazgo escasa (superior al 90\%), que es la mayoritaria. No obstante, el modelo no predice bien los casos de capacidad elevada de liderazgo.

A partir de los resultados obtenidos, se observa que las variables explicativas edad, género y procedencia no tienen ninguna incidencia en la adquisición de la capacidad de liderazgo. Las que sí influyen son las puntuaciones de cada uno de los factores, es decir, las competencias recogidas en estos factores. Más concretamente, las competencias que agrupa el primer factor (conocimientos básicos de la profesión, capacidad de aplicar conocimientos a la práctica, capacidad de análisis y síntesis, planificación y gestión

\footnotetext{
${ }^{3} \chi^{2}=69.901$, con un p-valor asociado de 0.0000 .

${ }^{4}$ El pseudo- $\mathrm{R}^{2}$ de McFadden es una medida relativa de la bondad de ajuste. Valores comprendidos entre 0,2 y 0,4 pueden considerarse como un buen ajuste (Hauber et al., 2016).
} 
del tiempo y capacidad de aprendizaje) repercuten positivamente en la probabilidad de que el estudiante considere tener bastante capacidad de liderazgo respecto a sentirse con una nula capacidad de liderazgo. Por lo que respecta a las competencias incluidas en el segundo factor (diseño y gestión de proyectos, habilidad para trabajar de manera autónoma, iniciativa y espíritu emprendedor, y comunicación oral y escrita en la propia lengua), influyen positivamente en el incremento de la probabilidad de sentirse con bastante (o con elevada) capacidad de liderazgo respecto a que el estudiante se considere con una nula capacidad. Lo mismo sucede con los factores 3 y 4, que recogen respectivamente las competencias críticas y comunicativas (capacidad de crítica y autocrítica, compromiso ético, pensamiento crítico, capacidad de comunicarse, capacidad de adaptación a nuevas situaciones y conocimiento de una segunda lengua), y las relacionadas con la toma de decisiones y el trabajo en equipo (toma de decisiones, trabajo en equipo, capacidad de generar nuevas ideas (creatividad), resolución de problemas).

Más concretamente, si nos fijamos en los valores que ofrecen los odd-ratio por factor, se aprecia que cuando la puntuación de cualquiera de los cuatro factores incrementa aumenta la probabilidad que el individuo se considere con un elevado liderazgo respecto a la probabilidad de que se sienta con nulo liderazgo. Del mismo modo, cuando la puntuación de los tres últimos factores sube, también lo hace la probabilidad de que el individuo se considere con un nivel de liderazgo medio respecto a la probabilidad de que se sienta con nulo liderazgo.

Estos resultados confirman la importancia que tiene la adquisición de las distintas competencias impartidas en el grado de enfermería a la hora de que los estudiantes valoren y ejerzan su capacidad de liderazgo. De ello puede derivarse la necesidad por parte de los académicos de transmitir de forma adecuada las competencias, así como de proceder correctamente y de forma precisa a su evaluación. Del grado de asimilación de estas habilidades por parte de los estudiantes dependerá en este caso concreto, como se ha visto, su capacidad de liderazgo y la visión que tienen de la misma.

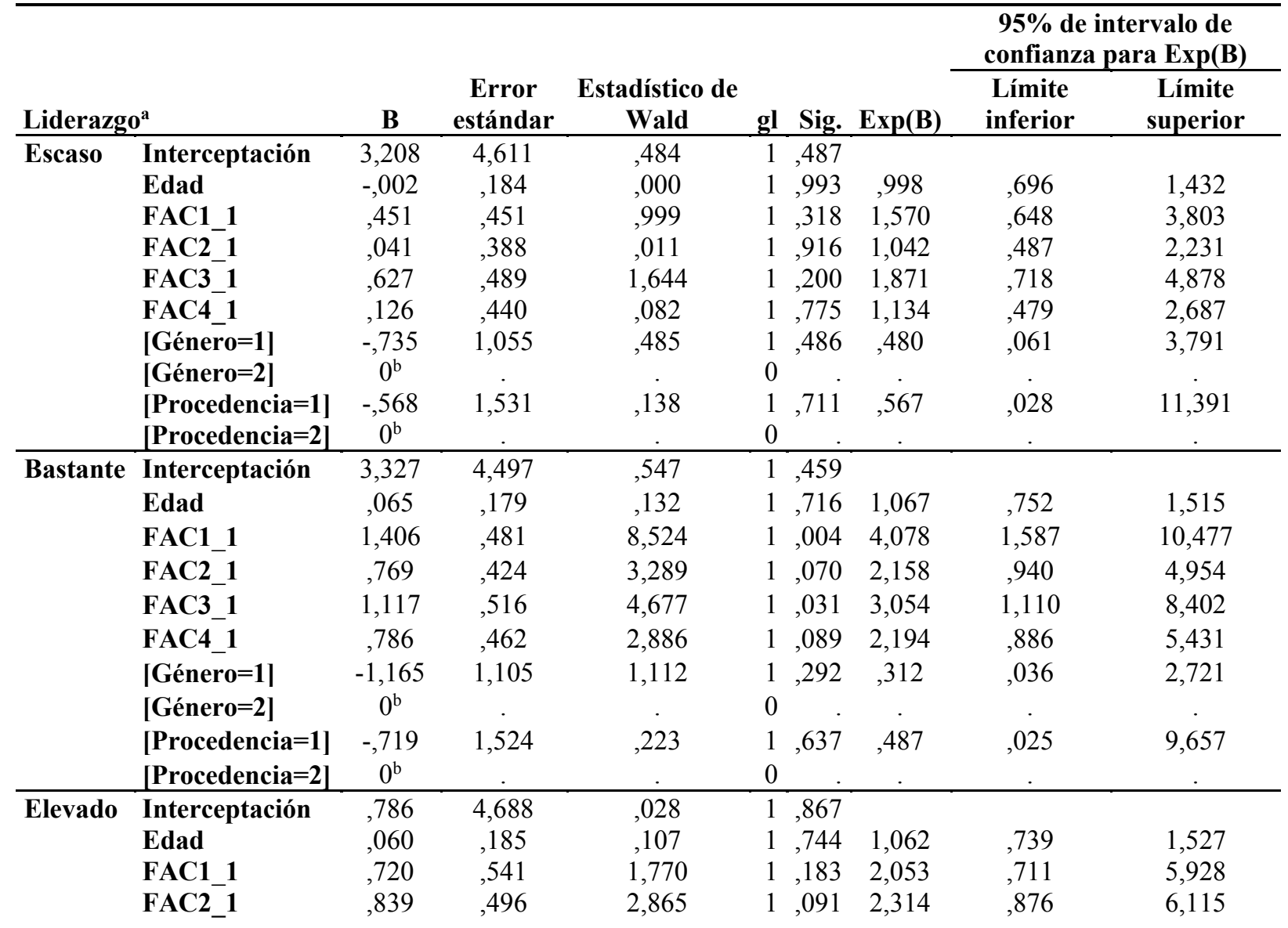




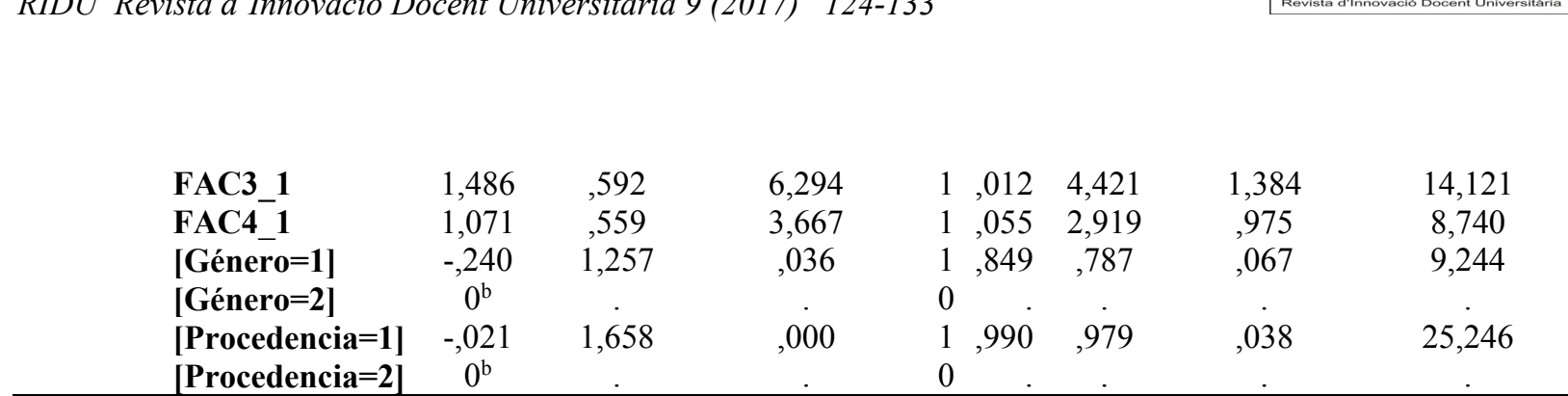

Notas. a. La categoría de referencia es: Nulo. b. Este parámetro está establecido en cero porque es redundante.

Tabla 5: Resultados del modelo logit multinomial para explicar la capacidad de liderazgo en los estudiantes de enfermería.

\section{Conclusiones}

El avance de la globalización ha contribuido a la rápida evolución de las distintas estructuras presentes en cualquier institución, obligando a diferenciar entre ser capaz y ser competente. En este sentido, el liderazgo no puede entenderse solamente como una acumulación de conocimientos sino también de habilidades y competencias. Esta evolución no está exenta de problemas dado que la capacidad de liderar involucra aspectos personales y humanos que lo dificultan, y dificultan también la definición del mismo en base a las competencias exigibles.

Así, un buen líder es capaz de trabajar tanto autónomamente como en grupo y de coordinarse con su equipo de trabajo propiciando un ambiente de trabajo crítico y responsable con respecto a la toma de decisiones. Pero está claro que todo ello dependerá del campo o área donde deba desempeñar sus responsabilidades. En el caso del campo sanitario, su compromiso con la ética profesional y la transmisión de la misma en todas sus acciones es básico, dado que le permite trascender, al igual que su capacidad organizativa y comunicativa, puesto que le ayudan a elaborar y transmitir sus conocimientos con el objetivo de mejorar el cuidado de los pacientes en la práctica clínica diaria. El hecho de saber transmitir de forma clara y precisa sus conocimientos, y que pueda comunicarse de manera directa y apropiada, brindará al paciente un mejor entendimiento de la situación, a la vez que se asegura la correcta interpretación del mensaje.

Para nuestro caso de estudio, centrado en el grado de enfermería, se observa cómo las competencias, que se han organizado alrededor de cuatro factores clave, la capacitación para la profesionalización, el trabajo autónomo, la capacidad de crítica y comunicación, y la capacidad de toma de decisiones y trabajo en equipo, ejercen una influencia positiva y significativa en la capacidad de liderazgo de los estudiantes, y en la probabilidad de incremento de esa capacidad. De ello se deduce la necesidad por parte de los docentes de trabajar en la adquisición de tales competencias de forma que al terminar los estudios, el nuevo profesional de la salud sepa orientar su profesión hacia las metas propuestas y lo haga con seguridad y responsabilidad, así como con capacidad de liderazgo.

\section{Agradecimientos}

Los datos de este trabajo pertenecen al proyecto: "La adquisición de competencias en los grados universitarios: visión y opinión de los estudiantes, docentes y empresarios (VIOPES)", financiado por la Universidad de Barcelona en la convocatoria de proyectos de investigación en Ciencias Sociales y Humanas 2012.

Las autoras también agradecen la ayuda recibida a través del proyecto REDICE16-1562: "Necessitats $i$ requeriments laborals i formació dels graduats. Anàlisi de les discrepàncies competencials a partir de les pràctiques empresarials", financiado por el Institut de Ciències de l'Educació (ICE), de la Universitat de Barcelona. 


\section{Referencias}

Alcañiz, M., Claveria, O., Riera-Prunera, C. (2014) Competencias en educación superior desde tres perspectivas diferentes: estudiantes, empleadores y académicos. Revista Iberoamericana de Educación (RIE), 66(2), pp. 1-19.

Alcañiz, M., Riera-Prunera, C., Claveria, O. (2013) La formació competencial dels llicenciats en economia i empresa: una visió des del seu entorn professional. Revista d'Innovació i Recerca en Educació, 6(2), pp. 64-85.

Buendía, L. (1994) Técnicas e instrumentos de recogida de datos. P. Colás y L. Buendía. Investigación educativa (pp. 201.244). Alfar, Sevilla.

CODEM (2016) Estilos de liderazgo en la gestión de Enfermería. Disponible en: http://www.codem.es/investigacion-2/estilos-de-liderazgo-en-gestion-de-enfermeria) (consultado 17 octubre de 2016).

Cortés-González, J., Hernández-Saavedra, M., Marchena-Rivera, T., Marqueti-Machado, M., Nava-Galán, M. (2012) Estilos de liderazgo en jefes de Servicio de Enfermería. Enf Neurol, 12(2), pp. 84-94.

Freire, M.J., Teijeiro ÁLvarez, M.M., Pais Montes, C. (2013) La adecuación entre las competencias adquiridas por los graduados y las requeridas por los empresarios. Revista de Educacion, 362, pp. 13-41.

González, J., Wagenaar, R. (2003) Tuning educational structures in Europe. Disponible en: http://eua.be/eua/jsp/en/upload/TUNING_Announcement_Closing_Conference.1084282515011.pdf (consultado 1 diciembre de 2016).

Hauber, A.B., González, J.M., Groothuis-Oudshoorn, C.G.M., Prior, T., Marshall, D.A., Cunningham, C., IJzerman, M.J. Bridges, J. (2016) Statistical methods for the analysis of discrete-choice experiments: a report of the ISPOR Conjoint Analysis Good Research Practices Task Force. Value in Health, 19(4), pp. 300-315.

Martín Del Peso, M., Rabadán-Gómez, A.B., Hernández-March, J. (2013) Desajustes entre formación y empleo en el ámbito de las enseñanzas técnicas universitarias: La visión de los empleadores de la Comunidad de Madrid. Revista de Educacion, 360, pp. 244-267.

Mason, G., Williams, G., Cranmer, S. (2009) Employability skills initiatives in higher education: what effects do they have on graduate labour market outcomes? Education Economics, 17(1), pp. 1-30.

Moreno-Arroyo, M.C. (2015) Bases para la adquisición de una cultura preventiva ante los riesgos biológicos en los estudiantes de Grado de Enfermería de la Universidad de Barcelona. TDX (Tesis Doctorals en Xarxa). Universitat de Barcelona.

Navarro, M.M., Iglesias, M.P., Torres, P.R. (2006) Las deficiencias formativas en la Educación Superior: El caso de las Ingenierías Educational Gaps in Higher Education: Analysis of the case of the Engineering Careers. Cuadernos de Gestión, 6(1), pp. 27-43.

Paadi, K. (2014) Perception on employability skills necessary to enhance human resource management. Graduates prospects of securing a relevant place in the labour market. European Scientific Journal, Special ed. (August), pp. $129-143$.

Teijeiro, M., Rungo, P., Freire, M.J. (2013) Graduate competencies and employability: The impact of matching firms' needs and personal attainments. Economics of Education Review, 34, pp. 286-295.

UB (2014) Memoria para la verificación del Título de Grado en Enfermería por la Universidad de Barcelona. 